\title{
Olanzapine in the Treatment of Behavioral Problems Associated with Autism: An Open-Label Trial in Kuwait
}

\author{
Abdullahi Fido $^{a}$ Samira Al-Saad ${ }^{b}$ \\ ${ }^{a}$ Department of Psychiatry, Faculty of Medicine, Health Sciences Centre, Kuwait University, and \\ ${ }^{\mathrm{b}}$ Kuwait Autism Center, Kuwait
}

\section{Key Words}

Autism - Olanzapine - Atypical antipsychotic agent •

Kuwait, clinical trial

\begin{abstract}
Objectives: To study the efficacy and safety of olanzapine for the treatment of children with autism associated with disruptive behavior problems. Subjects and Methods: A prospective open-label trial was conducted on 40 male children (mean age $12.2 \pm 2.2$ years, range 7-17 years) meeting Diagnostic Statistical Manual IV criteria for autism. After a washout period from previous medications (2-14 days), patients received olanzapine (5-10 $\mathrm{mg} /$ day) for a 13-week treatment period. The primary efficacy measures were Aberrant Behavior Checklist $(A B C)$ and Clinical Global Impressions-Severity (CGI-S) done at baseline and end of treatment. At the beginning and end of treatment, patients underwent laboratory and physical investigations: ECG, chest X-ray, urinalysis, serum chemistry, blood glucose and lipid profile, hematology and hepatitis B serology. Results: Paired comparison of baseline and 13-week endpoint scores showed significant reductions in $A B C$ subscale scores for irritability $(p<0.0001)$, lethargy ( $p<0.0001)$, stereotyped behavior ( $<<0.005)$, hyperactivity $(p<0.0001)$ and inappropriate speech $(p<0.005)$. Of 40 patients, 12 (30\%) were considered as 'improved' on CGI-S scores compared to baseline, a statistically significant difference $(p<0.05)$. No liver enzyme elevation or any other serum biochemical changes resulted from treatment, which
\end{abstract}

was not associated with significant body weight changes or any other treatment-emergent side effects. Conclusions: The study shows that olanzapine treatment can be beneficial in alleviating some behavioral symptoms (irritability, hyperactivity/noncompliance and lethargy/withdrawal) associated with autism. The short period of this trial limits inferences about adverse effects such as body weight increase and tardive dyskinesia. Further long-term placebocontrolled studies of olanzapine are required.

Copyright $\odot 2008$ S. Karger AG, Basel

\section{Introduction}

Autism is a lifelong, biologically based, neurobehavioral, multidimensional disorder that affects verbal and nonverbal communication, physical and verbal social interactions, and daily routines and activities. The Diagnostic Statistical Manual IV [1] defines the core criteria for autism as (a) poor shared social interaction, (b) impaired communication and imaginative play, and (c) very limited and narrowed interests and activities beginning before the age of 3 years. Autism, however, is also a heterogeneous disorder with each person having specific individual needs, deficits and strengths. The prevalence of autism is estimated at 3.4 per 1,000 children [2]. Unfortunately, there is little information available concerning the prevalence of autism in Kuwait, but the indications are that autism exists [3].

\section{KARGER}

Fax +4161306 1234

E-Mail karger@karger.ch

www.karger.com
(C) 2008 S. Karger AG, Basel

$1011-7571 / 08 / 0175-0415 \$ 24.50 / 0$

Accessible online at:

www.karger.com/mpp
Dr. Abdullahi Fido, MD, MRCPsych

Department of Psychiatry, Faculty of Medicine, Health Sciences Centre Kuwait University, PO Box 24923

Safat 13110 (Kuwait)

Tel. +965 533 0467, Fax +965 533 8904, E-Mail fido@hsc.edu.kw 
The nature and causes of autism still remain unresolved although there is a clear consensus that autism is genetically determined, and currently no specific gene has been identified [4-6]. Other studies have implicated gluten, a protein found in wheat and several other grains as the cause of autism $[7,8]$. The role of some neurotransmitter abnormalities and toxic trace element levels in the etiology of autism has been explored, but efforts have failed to prove a direct causative link [9-13].

There is no cure for autism as no single drug or therapy has proven effective for treating the core deficits of autism. Children and adolescents with autism can display disruptive behaviors such as hand flapping, hyperactivity, tantrums, aggression, head banging and other self-injurious behaviors. These types of behaviors have created challenges and barriers for teachers, parents and health care givers. Educational and behavioral therapies such as treatment of behavior by interruption of free operant behavior to reduce violent behavior associated with autism, and the use of errorless learning and backward chaining techniques for the acquisition of information skills in adults with autism are key components of treatment and may be effective in many cases [14-16]. However, when persistent maladaptive behaviors inhibit therapeutic progress, pharmacological therapy may be considered as a treatment option.

In numerous studies a variety of medications for reducing different behavioral problems in individuals with autism have been evaluated. Hyperactivity and attention deficit disorders in children with autism have been targeted by psychostimulants, and $\alpha$-adrenergic agonists such as clonidine $[17,18]$.

Conventional neuroleptics such as haloperidol have been used to treat the more aggressive behavior associated with autism; however, their side effects that include a high risk of tardive dyskinesia and withdrawal dyskinesia are considered unacceptable $[19,20]$. Hence, atypical antipsychotic drugs are being studied as off-label medications to treat disruptive behavior in subjects with autism because of their increased safety and efficacy over conventional neuroleptics.

Atypical antipsychotic agents, risperidone $[21,22]$ and olanzapine [23-25], which block postsynaptic dopamine and serotonin receptors, have been shown to be beneficial in the treatment of disruptive behavior in adult patients with schizophrenia and may be beneficial in children with autistic disorders who have similar behavioral disturbances. These studies with olanzapine involved a limited number of patients ranging from 8 to 25 .

Equally important, olanzapine has not been approved by the regulatory authorities in Arab countries for the treatment of children with autism pending prior local clinical trial. For this purpose, an open-label trial study was adopted in order to replicate previous findings of the clinical efficacy of olanzapine in the treatment of disruptive behavior associated with autism in Kuwait where alternative nonpharmacological treatments such as behavioral therapy modalities have not been established.

\section{Subjects and Methods}

Subjects

Forty male children from single-incidence autism families aged 7-17 years (mean age $12.2 \pm 2.2$ years) who were attending Kuwait Autism Center were included in the study. Currently, the center hosts 250 children and provides education for autistic children and children with other pervasive developmental disorders. The clinical diagnosis of autism was made by a panel of psychiatrists and clinical psychologists specialized in the field of autism and pervasive developmental disorders. The mean age of autism at diagnosis was $2.7 \pm 0.3$ years. All children were thoroughly examined with respect to mental state, social, neurodevelopmental and general medical status. Children with co-occurring medical conditions thought to be possibly etiologically related to autism such as tuberous sclerosis, neurofibromatosis, phenylketonuria, chromosomal anomaly identified through karyotyping, fragile-X syndrome or significant CNS injury, were excluded from the study. Children with concomitant schizophrenia or other psychotic disorders and those with a history of tardive dyskinesia, neuroleptic malignant syndrome, seizures within the previous 3 months or previous intolerance to olanzapine were also excluded from study. A prior written consent was obtained from each parent or caretaker after the procedures and possible side effects were explained to them. The patients were comparable with respect to socioeconomic status, severity of illness and level of cognitive functioning (IQ >70). The research protocol was approved by the Ethics Committee of Kuwait Autism Center.

\section{Pretrial Screening}

At both entry point and end of study (13 weeks later), children underwent laboratory and physical investigations including ECG, chest X-ray, urinalysis, serum chemistry, blood glucose, lipid profiles, hematology and hepatitis B serology. Baseline body weights of all children were measured before commencing olanzapine treatment. Clinical assessments were carried out initially at the screening visit followed by a baseline assessment after the washout period. Side effects such as extrapyramidal symptoms, akathisia and dyskinesia were further assessed by the Abnormal Involuntary Movement Scale [26] at baseline and 13 weeks. This is a 12 -item scale that rates the presence and severity of abnormal movement in three body areas: orofacial, extremities and trunk.

\section{Outcome Measures}

Each patient was assessed with the Aberrant Behavior Checklist (ABC) and Clinical Global Impressions-Severity (CGI-S) [27]. The $\mathrm{ABC}$ is a 58 -item checklist that gives a total composite and measures 6 areas of behavior: irritability, lethargy/withdrawal, stereotyped behavior, hyperactivity and inappropriate speech. 
The CGI-S is a 7-point rating scale ranging from 'normal' to 'among the most extremely ill patients'. The measurements were performed by a consultant psychiatrist (A.F.) and a clinical psychologist (S.A.) who had experience with ABC and CGI-S rating instruments.

\section{Study Design and Medications}

The trial consisted of two study periods: (a) a washout period from previous antipsychotic drugs of 2-9 days for oral antipsychotics and 14 days for depot antipsychotic medications, and (b) an open-label therapy period in which all patients started olanzapine therapy at $2.5 \mathrm{mg}$ twice daily for the first 7 days. Thereafter, investigators adjusted the daily dosage upwards or downwards by $2.5 \mathrm{mg}$ as needed with the maximum dosage limit of 10 $\mathrm{mg} / \mathrm{day}$, based on package labeling for olanzapine. The dosages ranged from 5 to $10 \mathrm{mg} /$ day with a mean of $7.5 \mathrm{mg} /$ day. The minimal criterion for efficacy was a $25 \%$ reduction from baseline for the $\mathrm{ABC}$ subscale scores and at least a 1-point improvement on the CGI-S scale.

\section{Data Analysis}

As an initial analysis, the degree of reliabililty between the two interraters was determined by the use of the interclass correlation coefficient, which was 0.60 , and hence the mean of the two ratings was used. The paired t test was used to compare the differences between the means of $\mathrm{ABC}$ scores before and after treatment. The Fisher exact test was used to examine the difference in the proportion of side effects before and after treatment. The statistically significant difference was set at $\mathrm{p} \leq 0.05$.

\section{Results}

All 40 autistic children completed the 13-week openlabel treatment with olanzapine. The mean subscale scores for $\mathrm{ABC}$ before and after olanzapine treatment are given in table 1. Paired comparisons of baseline and 13-week endpoint scores showed significant improvements over time for the ABC subscale scores of irritability $(p=0.0001)$, lethargy/withdrawal $(\mathrm{p}=0.0001)$, stereotyped behavior $(\mathrm{p}=0.005)$, hyperactivity $(\mathrm{p}=0.0001)$ and inappropriate speech $(\mathrm{p}<0.005)$. Of the 40 children, $12(30 \%)$ with a baseline CGI-S score of $4.8 \pm 0.6$ were considered as 'much improved' after olanzapine treatment (CGI-S score $3.6 \pm 0.5)$, and the difference was statistically significant $(\mathrm{p}<0.05)$. The number of patients $(4 ; 10 \%)$ with treatment-emergent extrapyramidal symptoms and dyskinetic symptoms assessed by the Abnormal Involuntary Movement Scale remained basically the same as at baseline (score $2.8 \pm 0.5$ ) and at the end of treatment (score $3.0 \pm 0.7)$. Neither significant hepatic enzyme elevation nor any serum chemistry changes were observed after treatment. The mean body weight was $52.5 \pm 3.5 \mathrm{~kg}$ before treatment and $52.75 \pm 3.1 \mathrm{~kg}$ after treatment, indicating no statistically significant body weight changes
Table 1. Mean subscale scores for the $A B C$ before and after treatment

\begin{tabular}{lrrl}
\hline ABC subscales & Before & After & p value \\
\hline Irritability & $20.0 \pm 8.1$ & $6.2 \pm 4.5$ & $<0.0001$ \\
Lethargy/withdrawal & $14.0 \pm 3.2$ & $4.7 \pm 4.4$ & $<0.0001$ \\
Stereotypic behavior & $8.2 \pm 5.0$ & $3.9 \pm 4.3$ & $<0.005$ \\
Hyperactivity/noncompliance & $28.0 \pm 9.5$ & $12.2 \pm 7.9$ & $<0.0001$ \\
Inappropriate speech & $3.7 \pm 3.0$ & $2.7 \pm 1.8$ & $<0.005$ \\
\hline
\end{tabular}

$\mathrm{p}=0.46)$. Only 5 of the 40 children $(12.5 \%)$ reported limited mild transient side effects such as drowsiness and sedation when treatment was initiated, but these were not present at the end of the 13-week treatment period.

\section{Discussion}

Autism is a particularly devastating disorder with no curative therapies currently available. Treatment focuses on targeting behavioral problems with medications. The primary finding is that open-label trial olanzapine is effective in the treatment of some symptoms (irritability, lethargy/withdrawal and hyperactivity/noncompliance) associated with autism as indicated by the decrease in $\mathrm{ABC}$ subscale scores, and in symptom severity improvement on the CGI-S score with transient mild side effects. Our findings are in partial agreement with those reported previously $[24,25,28]$. In the study of Kemner et al. [25] regarding the safety and efficacy of olanzapine in 25 children with autism, significant improvement was reported on three subscales of the ABC (irritability, hyperactivity and inappropriate speech), but no effect on the other symptoms. In Hollander et al. [28], a double-blind placebo-controlled pilot study of olanzapine on 16 children with pervasive developmental disorders, a significant clinical improvement in $50 \%$ of the olanzapine and $21 \%$ of the placebo group was reported. However, these studies also raised concerns about the risk of significant weight gain and other side effects associated with olanzapine treatment. However, in our study, only limited mild transient side effects were reported when treatment was initiated, but cleared by the end of the trial period. The lack of serious side effects in this study might be due to the smaller dosages used or because of the short treatment period.

While the general measure of efficacy (25\% reduction in $\mathrm{ABC}$ scores after treatment) is considered effective, the 
effect was even more prominent in the improvement of symptoms such as irritability, lethargy and hyperactivity because these symptoms are present in more than $50 \%$ of children who present with autism, treatment with olanzapine should be considered as part of a comprehensive treatment strategy for children with autism. The current findings are noteworthy because of the increased interest in this disorder in the Arab world and the relative lack of alternative behavioral intervention treatment resources available. Unfortunately, many unanswered questions remain about the use of olanzapine. It is unclear how the use of such long-term medication will affect brain function, learning and development of a young brain, which is continually undergoing changes in neurotransmitters and receptors. The short period of this trial limits inferences about adverse effects such as hyperlipidemia, hyperprolactinemia, weight gain and tardive dyskinesia.
This was an open-label, exploratory study; it did not assess long-term maintenance of response. More research, especially that involving a long-term randomized, double-blind, placebo-controlled trial, is needed to examine the safety and efficacy of olanzapine in the treatment of disruptive behaviors found in children with autism.

\section{Conclusion}

Olanzapine was effective and well tolerated for the treatment of some behavioral symptoms (irritability, hyperactivity/noncompliance and lethargy/withdrawal) associated with autism. The short period of this trial limits inference about common side effects such as weight gain, lipidemia and tardive dyskinesia.

\section{References}

1 American Psychiatric Association: Diagnostic and Statistical Manual of Mental Disorders, ed 4. Washington, American Psychiatric Association, 2002.

2 Frombonne E: Epidemiological surveys of autism and other pervasive developmental disorders: an update. J Autism Dev Disord 2003;33:365-382

3 The Annual Statistical Abstract. Kuwait Autism Society 2006;2:51-58.

4 Bailey A, Lecouteur A, Gottesman J: Autism as a strongly genetic disorder: evidence from a British twin study. Psychol Med 1995;25: 63-77.

5 Piven J, Palmer P, Jacobi D: The broader autism phenotype: evidence from a family study of multiple-incidence autism families. Am J Psychiatry 1997;154:185-190.

6 Sykes NH, Lamb JS: Autism: the quest for the genes. Expert Rev Mol Med 2007;9:1-15.

7 Whitely P: A gluten-free diet as an intervention for autism and associated spectrum disorders: preliminary findings. Autism 1999;3: $45-65$.

8 Murch S: Diet, immunity, and autistic spectrum disorders. J Pediatr 2005; 146:582584.

9 Janusonis S: Statistical distribution of blood serotonin as a predictor of early autistic brain abnormalties. Theor Biol Med Model 2007;19:2-27.

10 Fido A, Dashti H, Al-Saad S: Biological correlates of childhood autism: 'trace elements'. Trace Elem Electrol 2002;19:1-4.

-11 Fido A, Al-Saad S: Toxic trace elements in the hair of children with autism. Autism 2005;9: 290-298.
-12 Dosman CF, Brian JA, Drmic IE: Children with autism: effect of iron supplementation on sleep and ferritin. Pediatr Neurol 2007;36: 152-158.

13 Adams JB, Romdalvik J, Ramanujam VM: Mercury, lead, and zinc in baby teeth of children with autism versus controls. J Toxicol Environ Health 2007;70:1046-1051.

14 Horner RH, Carr EG, Strain PS: Problem behavior interventions for young children with autism: a research synthesis. J Autism Dev Disord 2002;32:423-446.

15 Hagopian LP, Pruzek JL, Bowman LG: Assessment and treatment of problem behavior occasioned by interruption of free-operant behavior. J Appl Behav Anal 2007;40:89103.

16 Jerome J, Frantino E, Sturmey P: The effects of errorless learning and backward chaining on the acquisition of internet skills in adults with developmental disabilities. J Appl Behav Anal 2007;40:185-189.

17 Posey DJ, McDougle CJ: The pharmacotherapy of target symptoms associated with autistic disorder and other pervasive developmental disorders. Harv Rev Psychiatry 2000; 8:45-63.

18 Aman MG: Management of hyperactivity and other acting-out problems in patients with autism spectrum disorder. Semin Pediatr Neurol 2004;11:225-228.

19 Malone R, Maislin G, Choudhury M: Risperidone treatment in children and adolescents with autism: short-and long-term safety and effectiveness. Am Acad Child Adolesc Psychiatry 2002;41:140-147.
20 McCracken J, Mcgough J, Shah B: Risperidone in children with autism and serious behavioral problems. N Engl J Med 2002;347: 314-321.

21 Padina GJ, Bossie CA, Yousef E: Risperidone improves symptoms in children with autism in a randomized, double-blind, placebocontrolled trial. Autism Dev Disord 2007;37: 367-373.

22 West L, Waldrop J: Risperidone use in the treatment of behavioral symptoms in children with autism. Pediatr Nursing 2006;32: 550-555.

23 Potenza MN, Holmes JP, Kanes SJ: Olanzapine treatment of children, adolescents, and adults with pervasive developmental disorders: an open-label pilot study. J Clin Psychopharmacol 1999;19:37-44.

24 Malone R, Cater J, Sheikh R: Olanzapine versus haloperidol in children with autistic disorder: an open pilot study. J Am Child Adolesc 2001;40:887-894

$\checkmark 25$ Kemner C, Willemsen S, Sophie H: Openlabel study of olanzapine in children with pervasive developmental disorder. J Clin Psychopharmacol 2002;22:455-460.

26 Barnes TR: A rating scale for drug-induced akathisia. Br J Psychiatry 1989;154:672676.

27 Aman MG, Singh NN, Stewart AW: The Aberrant Behavior Checklist: a behavior rating scale for the assessment of treatment effects. Am J Ment Defic 1985;89:485-491.

28 Hollander E, Wasserman S, Swanson EN: A double-blind placebo-controlled pilot study of olanzapine in childhood/adolescent pervasive developmental disorder. J Child Adolesc Psychopharmacol 2006;16:541-548. 